\author{
Military Technical College \\ Kobry El-Kobbah, \\ Cairo, Egypt
}

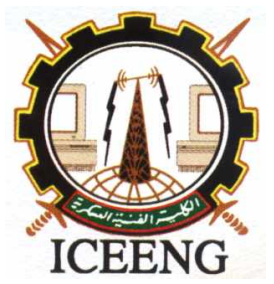

\author{
$6^{\text {th }}$ International Conference \\ on Electrical Engineering \\ ICEENG 2008
}

\title{
Diffraction problem by the system of strips in a plane waveguide
}

$$
\text { By }
$$

\author{
S. A. Pogarsky*
}

\section{Abstract:}

The eigenwaves diffraction problem in a plane waveguide by strips is considered. Strips are placed in the same plane. Waveguide is filled by non-ideal dielectric. The operator approach in combination with the spectral-domain approach is used. Paired integral equations for single strip are solved by the spectral-domain approach. The structure's properties in the whole with finite number of discontinuities are obtained by the operator approach by use the reflection and transmission operators of single strip. Dependencies of reflection and transmission coefficients as a function of strip length and distance between strips are represented. They allow to determine passbands and stopbands. The properties of such structures allow to create frequency selection devices with high level of parameters.

\section{Keywords:}

Plane waveguide, spectral domain approach, diffraction, strip

* V.N. Karazin Kharkov National University, Kharkov, Ukraine 


\section{Introduction:}

The diffraction problems by different discontinuities in rectangular waveguides are actual in modern electrodynamics. One can find enough investigations in which the different approaches are used. For example, the method suggested in [1] allows investigating the properties of rectangular waveguide with resonant loads such as periodical ridges in $\mathrm{E}$ - plane. The implementation of a hybrid integral-equation/vector finite-element method formulation applicable to inhomogeneous obstacle scattering in hollow waveguide, requiring discretization just of the obstacle, is presented in [2]. The waveguides with periodically filled dielectrical cells are examined in [3].

In the present work the operator approach [4] is used concerning to the analysis of plane waveguide eigenmodes diffraction by finite number of strips of infinity thickness.

\section{Key-problem by spectral-domain approach for a single strip:}

Let us examine structure which is represented in Fig.1. The waveguide walls and strips are infinity conductive. It is supposed that dielectric permittivity is formulated as $\mathrm{e}=\mathrm{e}-$ ie . The main idea of operator approach consists in the solution finding of diffraction problem of eigenwave by single strip. The transmission and reflection operators of single strip are used in operator equation which is written for the structure in the whole.

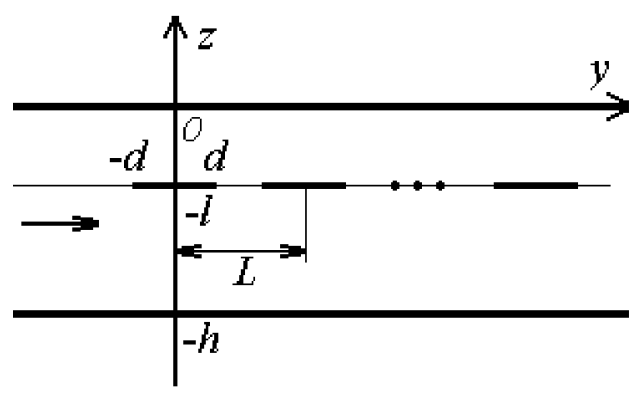

Figure (1): Coordinates system and structure geometry

For $\mathrm{H}$ - polarization let us represent the single non zero component of magnetic field in the form

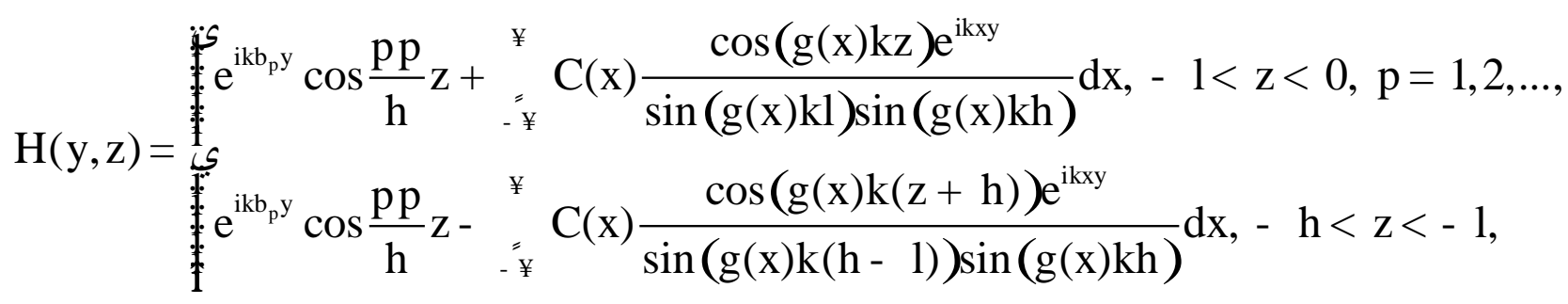


The unknown spectral function $\mathrm{C}(\mathrm{x})$ may be defined as follow

$$
\begin{aligned}
& 2^{¥} \underset{\sim}{¥} \mathrm{C}(\mathrm{x}) \frac{\mathrm{g}(\mathrm{x})}{\sin \mathrm{g}(\mathrm{x}) \mathrm{kh}} \mathrm{e}^{\mathrm{ikxy}} \mathrm{dx}=-\frac{2 \mathrm{pp}}{\mathrm{kh}} \mathrm{e}^{\mathrm{ikb_{p } y}} \sin \frac{\mathrm{ppl}}{\mathrm{h}},|\mathrm{y}|<\mathrm{d} \text {, } \\
& \stackrel{¥}{¥} \mathrm{C}(\mathrm{x}) \frac{1}{\sin (\mathrm{g}(\mathrm{x}) \mathrm{k}(\mathrm{h}-1)) \sin \mathrm{g}(\mathrm{x}) \mathrm{kl}} \mathrm{e}^{\mathrm{ikxy}} \mathrm{dx}=0,|\mathrm{y}|>\mathrm{d} \text {. }
\end{aligned}
$$

The system of linear algebraic equations may be obtained by the expanding of function $\mathrm{C}(\mathrm{x})$ in the series $\mathrm{C}(\mathrm{x})=-\mathrm{o}_{\mathrm{m}=1}^{\ddagger} \mathrm{C}_{\mathrm{m}} \mathrm{f}_{\mathrm{m}}(\mathrm{x}) \sin (\mathrm{g}(\mathrm{x}) \mathrm{kl}) \sin (\mathrm{g}(\mathrm{x}) \mathrm{k}(\mathrm{h}-1)) \quad[5]$, where $\mathrm{f}_{\mathrm{m}}(\mathrm{x})=\frac{\sqrt{2 \mathrm{~m}}}{\mathrm{x}} \mathrm{J}_{\mathrm{m}}(\mathrm{kdx}), \mathrm{m}=1,2, \ldots$ are the orthogonal basis. Thus the paired integral equations (2), (3) are equivalent to the system of equations

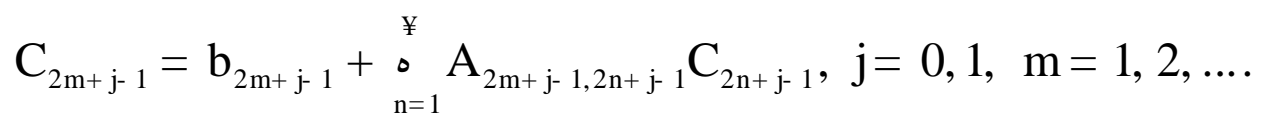

Integrands in expression (1) may have poles in the points $\beta_{s}$ which correspond to waveguide eigenwaves. After carrying out integration in the complex plane the expression for transmission and reflections coefficients may be represented as

$$
t_{p, p}=1-\frac{2 p^{2} i}{(k h)^{2}} \frac{(-1)^{p} p}{b_{p} \sin \frac{p l p}{h}} C\left(b_{p}\right), r_{p, p}=-\frac{2 p^{2} i}{(k h)^{2}} \frac{(-1)^{p} p}{b_{p} \sin \frac{p l p}{h}} C\left(-b_{p}\right), p=1,2, \ldots
$$

\section{Numerical results:}

Using the algorithm stated above the series of numerical simulation for transmission and reflection coefficients was realized. In Fig. 2 the dependencies of transmission coefficient (4) by the strip length of a single strip for different strip positions are represented. As one can see there are a lot of values of parameters which reduce the transmission coefficient to one or to zero approximately. Thus the structure may operate in passband or stopband regime. The positions of maximum and minimum values may be changed by strip position variation.

The dependences of reflection coefficient as a function of the distance between strips for the structure with four and ten strips depending on different dielectric loss e are presented in Fig.3. The reaction graphs show typical dependencies of stopbands depending on the discontinuities quantity. It is established that the increasing the 
quantity of discontinuities leads to these bands narrowing and to the appearance of oscillations in passband (the number of local minima is less then the number of discontinuities by one). When the distance between strips is a large value the reflection coefficient for the structure with finite quantity of strips approaches to the reflection coefficient of a single strip $\left(|\mathrm{r}|^{2}=0.5\right)$. It explained by the field attenuation and in this case strips interaction is insignificant.

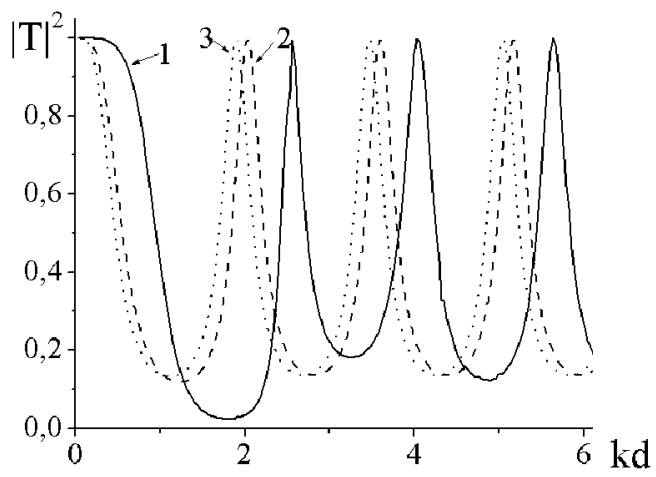

Figure (2): Dependencies of transmission coefficients for a single strip, $k h=3.2$.

$$
l-k l=0.32,2-k l=0.96,3-k l=1.6 \text {. }
$$

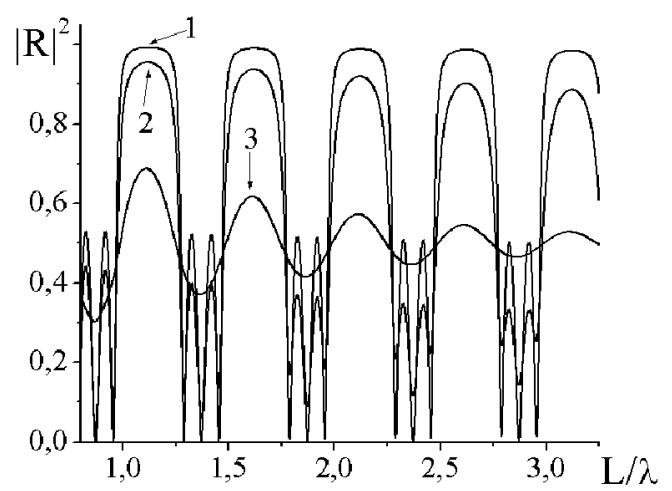

a)

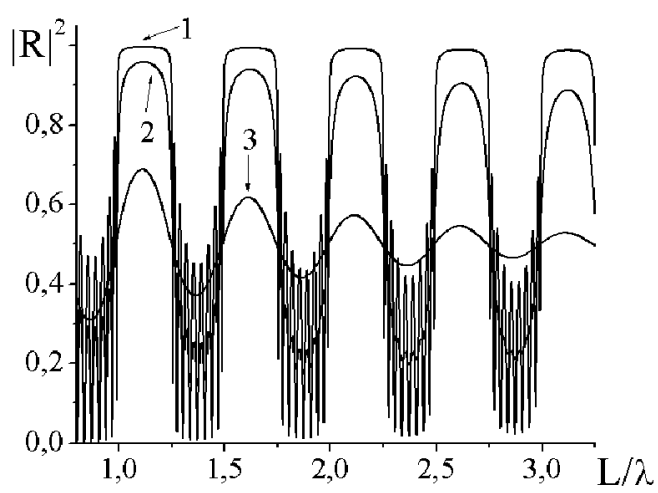

b)

Figure (3): Dependencies of reflection coefficients as a function of $L / 1$. a) four strips, b)ten strips. $k h=5, k d=1.2897, k l=0.5, \mathrm{e} \phi=11.2 .1-\mathrm{e} \phi=0.01,2-\mathrm{e} \phi=0.1,3-\mathrm{e} \phi=1$.

\section{Conclusions:}

The created approach which was applied to the solving diffraction problem of eigenwaves of plane waveguide by system of strips are universal enough. It may be explained by certain formalism of the procedure of total operator reflection finding if the reflection and transmission operators of single obstacle are known. This approach may be applied for solving any diffraction problems. 


\section{References:}

[1] G. Goussetis, Alexandros P. Feresidis, P. Kosmas, Efficient analysis, design, and filter applications of EBG waveguide with periodic resonant loads, IEEE Transactions on Microwave Theory and Techniques, Vol. 54, No. 11, P. 38853892, November 2006.

[2] R. H. Geschke, R. L. Ferrari, D. B. Davison, P. Meyer, The solution of weveguide scattering problems by application of an extended Huygens formulation, IEEE Transactions on Microwave Theory and Techniques, Vol. 54, No. 10, P. 36983705, October 2006.

[3] Hui Kan Liu, Tian Lin Dong, Propagation characteristics for periodic waveguide based on generalized conservation of complex power technique, IEEE Transactions on Microwave Theory and Techniques, Vol. 54, No. 9, P. 34793485, September 2006.

[4] L. N. Litvinenko, I. I. Reznick, D. L. Litvinenko, Wave diffraction by semiinfinite periodical structures, Proceedings of the Academy of Sciences of the Ukrainian SSR, No. 6, P. 62-66, June 1991.

[5] L. N. Litvininko, S. L. Prosvirnin, Transverse slot in a plane waveguide, Radiotehnika i Elektronika, Vol. 22, No. 7, P. 1321-1326, July 1977. 